\title{
La succession d'entreprise : une occasion de coopérer
}

\author{
Claude-André Guillotte, Anne-Marie Merrien, \\ Josée Charbonneau \& Étienne Fouquet \\ Institut de recherche et d'éducation pour les coopératives et \\ les mutuelles de l'Université de Sherbrooke (IRECUS)
}

\begin{abstract}
A vast proportion of Quebec's economic fabric is made up of small and medium-sized enterprises (SMEs). Recent studies underline the importance of ensuring the succession of these SMEs for the coming years. The collective option of cooperative takeover makes it possible to maintain the activities of these companies and to save the jobs they provide by selling the companies to employees or members of the community through conversion into cooperatives. However, this option has been largely neglected, which constitutes a missed opportunity for communities. Since 1980, 106 cooperatives have taken over a business in Quebec to ensure its continuity under this new status. The data show that, as of July 1 , $2021,96 \%$ of the cooperatives affected by these takeovers since 1980 were still in operation. This sustainability, including the maintenance of jobs and local economic activity, constitutes an argument in favour of exploring the cooperative model as an avenue for business takeovers.

\section{RÉSUMÉ}

Les petites et moyennes entreprises (PME) représentent la très grande majorité du tissu économique québécois. Des études récentes soulignent l'importance d'assurer la succession de ces PME dans les années à venir. L'option collective de la conversion en coopérative rend possible la continuité de ces entreprises et permet de sauver les emplois qu'elles offrent. Cette option de reprise implique le transfert des actifs et des activités de l'entreprise à ses employés ou à des membres de la communauté regroupés sous la formule juridique coopérative. Malheureusement, on a généralement négligé cette option, ce qui constitue une occasion manquée pour les communautés. Depuis 1980, pourtant, 106 coopératives ont repris une entreprise au Québec afin d'assurer sa pérennité sous ce nouveau statut. Les données montrent que, au 1 juillet 2021, 96\% des coopératives ayant subi de telles prises de contrôle existent encore. Cette durabilité, y compris la préservation d'emplois et de l'activité économique locale qui s'y rattachent, est une bonne raison d'explorer l'utilité du modèle coopératif pour la reprise d'entreprises.
\end{abstract}

Keywords / Mots clés : Business succession; Cooperative conversion / Reprise d'entreprises; Conversion en coopérative

La plupart des 1,2 million de petites et moyennes entreprises (PME) du Canada ont été touchées par la pandémie, ce qui aggrave les problèmes auxquels l'économie canadienne est confrontée depuis des années : le potentiel de fermetures 


\section{Guillotte, Merrien, Charbonneau, \& Fouquet (2021)}

permanentes et à grande échelle d'entreprises, en raison du nombre croissant de propriétaires approchant de la retraite, mais sans plan formel de relève de l'entreprise.

L'actualité récente fait d'ailleurs état du phénomène de la reprise de PME au Canada et plus particulièrement au Québec, dans le contexte du changement générationnel qui s'opère présentement. À titre d'exemple, on peut mentionner l'organisation du Sommet international du repreneuriat tenu à Montréal en novembre 2021, qui a rassemblé les acteurs majeurs de l'écosystème repreneurial du Québec et de l'international.

Le tissu économique québécois est composé en très grande majorité de PME, entreprises comptant moins de 500 employés $(99,8 \%$ du total d'après les données de 2019). Selon la plus récente étude de la Banque de développement du Canada $(B D C)^{1}$, près de 116000 entreprises canadiennes devront trouver une ou un repreneur.e dans les cinq prochaines années. Les propriétaires d'entreprise qui approchent l'âge de la retraite et qui doivent envisager passer le relais ne considèrent généralement que trois options : la transmission familiale, souvent privilégiée, la vente à des employés cadres ou la vente à un tiers privé. Or, une quatrième option mérite davantage d'attention : la reprise coopérative. Bon nombre de propriétaires de PME ne trouveront pas nécessairement la solution de reprise qui leur convient le mieux à travers les voies traditionnelles faute de préparation à la transmission, d'acquéreurs disponibles ou simplement d'un emplacement géographique adéquat.

L'option collective du repreneuriat coopératif permet de maintenir les activités de ces entreprises et de sauver les emplois qu'elles fournissent par la vente à des employés ou des membres de la communauté en les convertissant en coopératives. Cependant, cette option est grandement négligée, ce qui constitue une occasion manquée pour les communautés.

La reprise ou conversion coopérative concerne précisément l'intégration des activités économiques d'une entreprise au sein d'une autre entreprise constituée en coopérative. Depuis 1980, 106 coopératives ont repris une entreprise au Québec pour en assurer la continuité sous ce nouveau statut. La recension des données et la cartographie réalisées dans le cadre du projet CoopConvert révèlent que pour le Québec, en date du 1er juillet 2021,96\% des coopératives concernées par ces reprises depuis 1980 étaient toujours en activité. Cette durabilité, conjuguée aux autres effets directs de la reprise coopérative que représente le maintien des emplois et de l'activité économique locale, constituent des arguments qui militent en faveur de l'exploration du modèle coopératif comme piste de reprise d'entreprise.

Concrètement, de quel type de coopérative est-il question? Voici trois cas de figure. D'abord, il peut s'agir d'une reprise par les consommateurs dans le cas, par exemple, d'une entreprise funéraire ou d'une épicerie locale. Dans le cas des résidences funéraires, la Fédération des coopératives funéraires du Québec ${ }^{2}$ travaille activement à racheter et convertir des maisons funéraires privées en coopératives au bénéfice des communautés. On compte près de 100 points de services. Dans l'alimentation, il existe actuellement au Québec 70 coopératives de services marchands de proximité. Ces organisations sont implantées dans des communautés qui ont choisi d'assurer collectivement leurs services d'épicerie, de distribution d'essence, de quincaillerie, ou encore de comptoir de la Société des alcools du Québec (SAQ) par l'entremise d'une coopérative. Les citoyens et entreprises locales peuvent en devenir membres, de même que, dans certains cas, les travailleurs et des acteurs locaux. Par la coopérative de consommateurs (ou de solidarité), la communauté s'assure de conserver des services de proximité nécessaires à la vitalité des communautés.

Ensuite, il peut s'agir d'une reprise par les travailleurs de l'entreprise cédée. La coopérative de travail peut, par exemple, assurer la pérennité de l'entreprise dans le cas d'un restaurant-café ou encore d'une entreprise de services-conseils. Elle permet aux travailleurs de conserver leur emploi, tout en valorisant leur savoir-faire et leur vision entrepreneuriale. La vague de conversions coopératives des entreprises de services ambulanciers dans les années 1990-2000 fait foi du 


\section{Guillotte, Merrien, Charbonneau, \& Fouquet (2021)}

potentiel de la reprise d'entreprise par les travailleurs. Le secteur manufacturier offre aussi de belles occasions de reprise coopérative, comme c'est le cas de Promoplatik ${ }^{3}$ dans le Bas Saint-Laurent où les travailleurs ont repris la division imprimerie d'une entreprise plus large.

Finalement, des fournisseurs d'une entreprise peuvent également la reprendre sous la forme d'une coopérative de producteurs afin de maintenir leur accès aux produits et services offerts et de s'assurer d'un plus grand contrôle sur certains maillons de la chaîne de valeur. C'est notamment ce qu'ont choisi de faire des pomiculteurs et des agriculteurs en se regroupant et devenant des producteurs-membres de la Coopérative pomicole du Lac des Deux-Montagnes. ${ }^{4}$

Ainsi, dans un contexte où plusieurs propriétaires de PME cherchent à prendre leur retraite moyennant la vente à un juste prix, tout en pérennisant leur vision entrepreneuriale et en préservant les emplois, le modèle coopératif représente une option intéressante. La reprise coopérative, c'est aussi une forme de garantie que les activités des entreprises demeureront au Canada, évitant un déplacement de la propriété des PME vers des investisseurs étrangers. Que ce soit pour maintenir l'offre de produits et services au sein d'une communauté, pour préserver des emplois ou, tout simplement, pour conserver la propriété locale des entreprises, la reprise coopérative est sans aucun doute une option à considérer.

II existe une panoplie d'acteurs susceptibles de faciliter la reprise coopérative au Québec. Nommons, parmi tant d'autres, la Coopérative de développement régional du Québec (CDRQ) et le Centre de transfert d'entreprise du Québec (CTEQ $)^{6}$, qui ont tous deux développé une expertise importante en développement coopératif. Leurs équipes savent accompagner les propriétaires cédants et les groupes de repreneurs pour assurer la pérennité de nos PME. En complément, ces acteurs disposent d'une connaissance fine des programmes financiers disponibles pour la reprise d'entreprise sous la forme coopérative. Ces financements sont mis sur pied par plusieurs acteurs membres d'un écosystème plus large travaillant conjointement et conformément à la réalité des coopératives : le Mouvement Desjardins, ${ }^{7}$ la Caisse d'économie solidaire ${ }^{8}$ le Conseil québécois de la coopération et de la mutualité, ${ }^{9}$ Fondaction, ${ }^{10}$ le Fonds de solidarité $\mathrm{FTQ}{ }^{11}$ ou encore Investissement Québec ${ }^{12}$ et la Fiducie du Chantier de l'économie sociale. ${ }^{13}$

Comme un processus de transfert d'entreprise peut prendre entre 2 et 8 ans et vu le grand nombre d'entreprises qui chercheront preneurs au cours des prochaines années, il y a urgence d'agir. Plusieurs voies doivent être empruntées. D'abord, mentionnons l'éducation à la coopération auprès des groupes de travailleurs et syndicats, des ressources entrepreneuriales et des programmes collégiaux et universitaires en entrepreneuriat. Ensuite, accentuons la formation et l'information auprès des conseillers d'affaires (comptables, juristes, etc.). Finalement, suscitons l'éveil du grand public à l'égard du risque de voir le tissu économique de PME s'effriter. Les statistiques parlent d'elles-mêmes. Considérons la succession d'entreprise comme une occasion de coopérer pour bâtir des entreprises et des communautés durables.

\section{NOTES}

1. https://www.bdc.ca/fr/a-propos/analyses-recherche/acquisition-entreprise

2. https://www.fcfq.coop/

3. https://www.promoplastik.com/

4. http://www.naturpac.com/coop.html

5. https://cdrq.coop/la-reprise-collective-dune-entreprise

6. https://ctequebec.com/programmes/reprise-collective/

7. https://www.capitalregional.com/

8. https://caissesolidaire.coop/

9. https://www.cqcm.coop/

10. https://www.fondaction.com/ 


\section{Guillotte, Merrien, Charbonneau, \& Fouquet (2021)}

11. https://www.fondsftq.com/

12. https://www.investquebec.com/quebec/fr

13. http://fiducieduchantier.qc.cal

\section{ABOUT THE AUTHORS / LES AUTEURS}

Claude-André Guillotte est directeur de l'Institut de recherche et d'éducation pour les coopératives et les mutuelles de I'Université de Sherbrooke (IRECUS). Courriel : claude-andre.guillotte@usherbrooke.ca .

Anne-Marie Merrien est professionnelle de recherche à l'Institut de recherche et d'éducation pour les coopératives et les mutuelles de l'Université de Sherbrooke (IRECUS). Courriel : anne-marie.merrien@usherbrooke.ca .

Josée Charbonneau est professionnelle de recherche à l'Institut de recherche et d'éducation pour les coopératives et les mutuelles de l'Université de Sherbrooke (IRECUS). Courriel : josee.charbonneau2@usherbrooke.ca .

Étienne Fouquet est doctorant en Administration des affaires associé à l'Institut de recherche et d'éducation pour les coopératives et les mutuelles de l'Université de Sherbrooke (IRECUS). Courriel : etienne.fouquet@usherbrooke.ca . 